Groups Geom. Dyn. 5 (2011), 787-803

DOI $10.4171 / \mathrm{GGD} / 148$
Groups, Geometry, and Dynamics

(C) European Mathematical Society

\title{
Quasi-isometries of rank one $S$-arithmetic lattices
}

\author{
Kevin Wortman*
}

\begin{abstract}
We complete the quasi-isometric classification of irreducible lattices in semisimple Lie groups over nondiscrete locally compact fields of characteristic zero by showing that any quasi-isometry of a rank one $S$-arithmetic lattice in a semisimple Lie group over nondiscrete locally compact fields of characteristic zero is a finite distance in the sup-norm from a commensurator.
\end{abstract}

Mathematics Subject Classification (2010). 20F65, 20G30, 22E40.

Keywords. Quasi-isometry, arithmetic groups.

\section{Introduction}

Throughout we let $K$ be an algebraic number field, $V_{K}$ the set of all inequivalent valuations on $K$, and $V_{K}^{\infty} \subseteq V_{K}$ the subset of Archimedean valuations. We will use $S$ to denote a finite subset of $V_{K}$ that contains $V_{K}^{\infty}$, and we write the corresponding ring of $S$-integers in $K$ as $\mathcal{O}_{S}$. In this paper, $\boldsymbol{G}$ will always be a connected noncommutative absolutely simple algebraic $K$-group.

1.1. Commensurators. For any valuation $v \in V_{K}$, we let $K_{v}$ be the completion of $K$ with respect to $v$. For any set of valuations $S^{\prime} \subseteq V_{K}$, we define

$$
G_{S^{\prime}}=\prod_{v \in S^{\prime}} \boldsymbol{G}\left(K_{v}\right)
$$

and identify $\boldsymbol{G}\left(\mathcal{O}_{S}\right)$ as a discrete subgroup of $G_{S}$ using the diagonal embedding.

We let $\operatorname{Aut}\left(G_{S}\right)$ be the group of topological group automorphisms of $G_{S}$. An automorphism $\psi \in \operatorname{Aut}\left(G_{S}\right)$ commensurates $\boldsymbol{G}\left(\mathcal{O}_{S}\right)$ if $\psi\left(\boldsymbol{G}\left(\mathcal{O}_{S}\right)\right) \cap \boldsymbol{G}\left(\mathcal{O}_{S}\right)$ is a finite index subgroup of both $\psi\left(\boldsymbol{G}\left(\mathcal{O}_{S}\right)\right)$ and $\boldsymbol{G}\left(\mathcal{O}_{S}\right)$.

We define the commensurator group of $\boldsymbol{G}\left(\mathcal{O}_{S}\right)$ to be the subgroup of $\operatorname{Aut}\left(G_{S}\right)$ consisting of automorphisms that commensurate $\boldsymbol{G}\left(\mathcal{O}_{S}\right)$. This group is denoted as $\operatorname{Comm}_{\text {Aut }\left(G_{S}\right)}\left(\boldsymbol{G}\left(\mathcal{O}_{S}\right)\right)$. Notice that it differs from the standard definition of the

\footnotetext{
${ }^{*}$ Supported in part by an N.S.F. Postdoctoral Fellowship.
} 
commensurator group of $\boldsymbol{G}\left(\mathcal{O}_{S}\right)$ in that we have not restricted ourselves to inner automorphisms.

1.2. Quasi-isometries. For constants $L \geq 1$ and $C \geq 0$, an $(L, C)$ quasi-isometric embedding of a metric space $X$ into a metric space $Y$ is a function $\phi: X \rightarrow Y$ such that for any $x_{1}, x_{2} \in X$ :

$$
\frac{1}{L} d\left(x_{1}, x_{2}\right)-C \leq d\left(\phi\left(x_{1}\right), \phi\left(x_{2}\right)\right) \leq L d\left(x_{1}, x_{2}\right)+C .
$$

We call $\phi$ an $(L, C)$ quasi-isometry if $\phi$ is an $(L, C)$ quasi-isometric embedding and there is a number $D \geq 0$ such that every point in $Y$ is within distance $D$ of some point in the image of $X$.

1.3. Quasi-isometry groups. For a metric space $X$, we define the relation $\sim$ on the set of functions $X \rightarrow X$ by $\phi \sim \psi$ if

$$
\sup _{x \in X} d(\phi(x), \psi(x))<\infty .
$$

In this paper we will call two functions equivalent if they are related by $\sim$.

For a finitely generated group with a word metric $\Gamma$, we form the set of all quasiisometries of $\Gamma$, and denote the quotient space modulo $\sim$ by $\mathcal{Q} \mathcal{I}(\Gamma)$. We call $\mathcal{Q} \mathcal{I}(\Gamma)$ the quasi-isometry group of $\Gamma$ as it has a natural group structure arising from function composition.

1.4. Main result. In this paper we show:

Theorem 1.4.1. Suppose $K$ is an algebraic number field, $\boldsymbol{G}$ is a connected noncommutative absolutely simple algebraic $K$-group, $S$ properly contains $V_{K}^{\infty}$, and $\operatorname{rank}_{K}(\boldsymbol{G})=1$. Then there is an isomorphism

$$
\mathcal{Q I}\left(\boldsymbol{G}\left(\mathcal{O}_{S}\right)\right) \cong \operatorname{Comm}_{\mathrm{Aut}\left(G_{S}\right)}\left(\boldsymbol{G}\left(\mathcal{O}_{S}\right)\right) .
$$

Two special cases of Theorem 1.4.1 had been previously known: Taback proved it when $\boldsymbol{G}\left(\mathcal{O}_{S}\right)$ is commensurable to $\mathrm{PGL}_{2}(\mathbb{Z}[1 / p])$ where $p$ is a prime number $[\mathrm{Ta}]$ (we shall use Taback's theorem in our proof), and it was proved when $\operatorname{rank}_{K_{v}}(\boldsymbol{G}) \geq 2$ for all $v \in S$ by the author in [Wo1].

Examples of $S$-arithmetic groups for which Theorem 1.4.1 had been previously unknown include when $\boldsymbol{G}\left(\mathcal{O}_{S}\right)=\mathrm{PGL}_{2}(\mathbb{Z}[1 / m])$ where $m$ is composite. Theorem 1.4.1 in this case alone was an object of study; see Taback-Whyte [Ta-Wh] for their program of study. Theorem 2.4.1 below presents a short proof of this case. 
1.5. Quasi-isometry groups of non-cocompact irreducible $S$-arithmetic lattices. Combining Theorem 1.4.1 with the results from Schwartz, Farb-Schwartz, Eskin, Farb, Taback, and Wortman ([Sch1], [Fa-Sch], [Sch2], [Es], [Fa], [Ta], and [Wo1]) we have

Theorem 1.5.1. Suppose $K$ is an algebraic number field, and $\boldsymbol{G}$ is a connected, noncommutative, absolutely simple, $K$-isotropic, algebraic $K$-group. If either $K \nsubseteq \mathbb{Q}$, $S \neq V_{K}^{\infty}$, or $\boldsymbol{G}$ is not $\mathbb{Q}$-isomorphic to $\mathrm{PGL}_{2}$, then there is an isomorphism

$$
\mathcal{Q} \mathcal{I}\left(\boldsymbol{G}\left(\mathcal{O}_{S}\right)\right) \cong \operatorname{Comm}_{\mathrm{Aut}\left(G_{S}\right)}\left(\boldsymbol{G}\left(\mathcal{O}_{S}\right)\right) .
$$

Note that Theorem 1.5.1 identifies the quasi-isometry group of any non-cocompact irreducible $S$-arithmetic lattice in a semisimple Lie group over nondiscrete locally compact fields of characteristic 0 that is not virtually free.

Indeed, the condition that $\boldsymbol{G}$ is $K$-isotropic is equivalent to $\boldsymbol{G}\left(\mathcal{O}_{S}\right)$ being noncocompact, and it is well known that $\boldsymbol{G}\left(\mathcal{O}_{S}\right)$ is virtually free only when $K \cong \mathbb{Q}$, $S=V_{K}^{\infty}$, and $\boldsymbol{G}$ is $\mathbb{Q}$-isomorphic to $\mathrm{PGL}_{2}$. In this case, $\boldsymbol{G}\left(\mathcal{O}_{S}\right)$ is commensurable with $\mathrm{PGL}_{2}(\mathbb{Z})$.

1.6. Cocompact case. By Kleiner-Leeb [K-L], the classification of quasi-isometries of cocompact $S$-arithmetic lattices reduces to the classification of quasi-isometries of real and $p$-adic simple Lie groups. This classification is known; see e.g. [Fa] for an account of the cases not covered in [K-L].

1.7. Function fields. Our proof of Theorem 1.4 .1 also applies when $K$ is a global function field when we add the hypothesis that there exists $v, w \in S$ such that $\operatorname{rank}_{K_{v}}(\boldsymbol{G})=1$ and $\operatorname{rank}_{K_{w}}(\boldsymbol{G}) \geq 2$.

For more on what is known about the quasi-isometry groups of arithmetic groups over function fields - and for a conjectural picture of what is unknown - see [Wo2].

1.8. Outline of the proof and the paper. We begin Section 2 with a sort of largescale reduction theory. We examine a metric neighborhood, $N$, of an orbit of an $S$ arithmetic group, $\Gamma$, inside the natural product of Euclidean buildings and symmetric spaces, $X$. In Section 2.2 we show that the fibers of $N$ under projections to building factors of $X$ are geometric models for $S$-arithmetic subgroups of $\Gamma$.

In Section 2.3, we apply results from [Wo1] to extend a quasi-isometry $\phi: \Gamma \rightarrow \Gamma$ to the space $X$ that necessarily preserves factors.

Our general approach to proving Theorem 1.4.1 is to restrict $\phi$ to factors of $X$ and use the results from Section 2.2 to decompose $\phi$ into quasi-isometries of $S$ arithmetic subgroups of $\Gamma$. Once each of these "sub-quasi-isometries" is understood, they are pieced together to show that $\phi$ is a commensurator. An easy example of this technique is given in Section 2.4 by $\mathrm{PGL}_{2}(\mathbb{Z}[1 / m])$. We then treat the general case of Theorem 1.4.1 in Section 2.5 
Our proof in Section 2.5 relies on the structure of horoballs for $S$-arithmetic groups associated to the product of a symmetric space and a single tree. We prove the results that we need for these horoballs (that they are connected, pairwise disjoint, and reflect a kind of symmetry in factors) in Section 3, so that Section 3 is somewhat of an appendix. The proof is organized in this way because, in the author's opinion, it just makes it easier to digest the material. There would be no harm though in reading Section 3 after Section 2.4 and before Section 2.5 for those who prefer a more linear presentation.

Acknowledgements. I was fortunate to have several conversations with Kevin Whyte on the contents of this paper, and am thankful for those. In particular, he brought to my attention that $\mathrm{SL}_{2}(\mathbb{Z})$ is the only non-cocompact arithmetic lattice in $\mathrm{SL}_{2}(\mathbb{R})$ up to commensurability.

I am also grateful to the following mathematicians who contributed to this paper: Tara Brendle, Kariane Calta, Indira Chatterji, Alex Eskin, Benson Farb, Dan Margalit, Dave Witte Morris, and Jennifer Taback.

\section{Proof of Theorem 1.4.1}

Let $\boldsymbol{G}, K$, and $S$ be as in Theorem 1.4.1, and let $\phi: \boldsymbol{G}\left(\mathcal{O}_{S}\right) \rightarrow \boldsymbol{G}\left(\mathcal{O}_{S}\right)$ be a quasiisometry.

2.1. Geometric models. For each valuation $v$ of $K$, we let $X_{v}$ be the symmetric space or Euclidean building corresponding to $\boldsymbol{G}\left(K_{v}\right)$. If $S^{\prime}$ is a finite set of valuations of $K$, we let

$$
X_{S^{\prime}}=\prod_{v \in S^{\prime}} X_{v} .
$$

Recall that there is a natural inclusion of topological groups $\operatorname{Aut}\left(G_{S^{\prime}}\right) \hookrightarrow \operatorname{Isom}\left(X_{S^{\prime}}\right)$.

Let $\mathcal{O}$ be the ring of integers in $K$, and fix a connected subspace $\Omega_{V_{K}^{\infty}} \subseteq X_{V_{K}^{\infty}}$ that $\boldsymbol{G}(\mathcal{O})$ acts cocompactly on. Let $D_{\infty} \subseteq X_{V_{K}^{\infty}}$ be a fundamental domain for this action.

For each non-Archimedean valuation $w \in S-V_{K}^{\infty}$, we denote the ring of integers in $K_{w}$ by $\mathcal{O}_{w}$. The group $\boldsymbol{G}\left(\mathcal{O}_{w}\right)$ is bounded in $\boldsymbol{G}\left(K_{w}\right)$, so $\boldsymbol{G}\left(\mathcal{O}_{w}\right)$ fixes a point $x_{w} \in X_{w}$. We choose a bounded set $D_{w} \subseteq X_{w}$ containing $x_{w}$ with $\boldsymbol{G}\left(\mathcal{O}_{S}\right) D_{w}=$ $X_{w}$ and such that $g x_{w} \in D_{w}$ for $g \in \boldsymbol{G}\left(\mathcal{O}_{S}\right)$ implies that $g x_{w}=x_{w}$.

For any set of valuations $S^{\prime}$ satisfying $V_{K}^{\infty} \subseteq S^{\prime} \subseteq S$, we define the space

$$
\Omega_{S^{\prime}}=\boldsymbol{G}\left(\mathcal{O}_{S^{\prime}}\right)\left(D_{\infty} \times \prod_{w \in S^{\prime}-V_{K}^{\infty}} D_{w}\right) .
$$

Note that $\Omega_{S^{\prime}}$ is a subspace of $X_{S^{\prime}}$.

We endow $\Omega_{S^{\prime}}$ with the path metric. Since $\boldsymbol{G}\left(\mathcal{O}_{S^{\prime}}\right)$ acts cocompactly on $\Omega_{S^{\prime}}$, we have the following observation: 
Lemma 2.1.1. For $V_{K}^{\infty} \subseteq S^{\prime} \subseteq S$, the space $\Omega_{S^{\prime}}$ is quasi-isometric to the group $\boldsymbol{G}\left(\mathcal{O}_{S^{\prime}}\right)$.

2.2. Fibers of projections to buildings are $S$-arithmetic. In the large-scale, the fibers of the projection of $\Omega_{S}$ onto building factors of $X_{S}$ are also $S$-arithmetic groups (or more precisely, $S^{\prime}$-arithmetic groups). This is the statement of Lemma 2.2.2 below, but we will start with a proof of a special case.

Lemma 2.2.1. The Hausdorff distance between

$$
\Omega_{S} \cap\left(X_{S^{\prime}} \times \prod_{w \in S-S^{\prime}}\left\{x_{w}\right\}\right)
$$

and

$$
\Omega_{S^{\prime}} \times \prod_{w \in S-S^{\prime}}\left\{x_{w}\right\}
$$

is finite.

Proof. There are three main steps in this proof.

First, if $y \in \Omega_{S^{\prime}}$, then $y=g d$ for some $g \in \boldsymbol{G}\left(\mathcal{O}_{S^{\prime}}\right)$ and some

$$
d \in D_{\infty} \times \prod_{w \in S^{\prime}-V_{K}^{\infty}} D_{w} .
$$

Since $\boldsymbol{G}\left(\mathcal{O}_{S^{\prime}}\right) \leq \boldsymbol{G}\left(\mathcal{O}_{w}\right)$ for all $w \in S-S^{\prime}$, it follows from our choice of the points $x_{w}$ that

$$
\{y\} \times \prod_{w \in S-S^{\prime}}\left\{x_{w}\right\}=g\left(\{d\} \times \prod_{w \in S-S^{\prime}}\left\{x_{w}\right\}\right) \subseteq \Omega_{S} .
$$

Therefore,

$$
\Omega_{S^{\prime}} \times \prod_{w \in S-S^{\prime}}\left\{x_{w}\right\} \subseteq \Omega_{S} \cap\left(X_{S^{\prime}} \times \prod_{w \in S-S^{\prime}}\left\{x_{w}\right\}\right) .
$$

Second, we suppose that

$$
\{y\} \times \prod_{w \in S-S^{\prime}}\left\{x_{w}\right\} \subseteq \Omega_{S}
$$

for some $y \in X_{S^{\prime}}$. Then there exists a $g \in \boldsymbol{G}\left(\mathcal{O}_{S}\right)$ such that

$$
g y \in D_{\infty} \times \prod_{w \in S^{\prime}-V_{K}^{\infty}} D_{w}
$$

and $g x_{w} \in D_{w}$ for all $w \in S-S^{\prime}$. Notice that our choice of $D_{w}$ implies $g x_{w}=x_{w}$ for all $w \in S-S^{\prime}$. Thus, $g$ is contained in the compact group

$$
H_{w}=\left\{h \in \boldsymbol{G}\left(K_{w}\right) \mid h x_{w}=x_{w}\right\}
$$


for all $w \in S-S^{\prime}$. Consequently, g is contained in the discrete group

$$
\boldsymbol{G}\left(\mathcal{O}_{S}\right) \cap\left(G_{S^{\prime}} \times \prod_{w \in S-S^{\prime}} H_{w}\right) .
$$

We name this discrete group $\Gamma_{S^{\prime}}$.

Note that we have shown

$$
\{y\} \times \prod_{w \in S-S^{\prime}}\left\{x_{w}\right\} \subseteq \Gamma_{S^{\prime}}\left(D_{\infty} \times \prod_{w \in S-V_{K}^{\infty}} D_{w}\right) .
$$

Therefore,

$$
\Omega_{S} \cap\left(X_{S^{\prime}} \times \prod_{w \in S-S^{\prime}}\left\{x_{w}\right\}\right) \subseteq \Gamma_{S^{\prime}}\left(D_{\infty} \times \prod_{w \in S-V_{K}^{\infty}} D_{w}\right) .
$$

Third, we recall that

$$
\boldsymbol{G}\left(\mathcal{O}_{S^{\prime}}\right)=\boldsymbol{G}\left(\mathcal{O}_{S}\right) \cap\left(G_{S^{\prime}} \times \prod_{w \in S-S^{\prime}} \boldsymbol{G}\left(\mathcal{O}_{w}\right)\right)
$$

and use the definition of $\Gamma_{S^{\prime}}$ coupled with the fact that $\boldsymbol{G}\left(\mathcal{O}_{w}\right) \leq H_{w}$ to see that $\Gamma_{S^{\prime}}$ contains $\boldsymbol{G}\left(\mathcal{O}_{S^{\prime}}\right)$. Since, $\Gamma_{S^{\prime}}$ and $\boldsymbol{G}\left(\mathcal{O}_{S^{\prime}}\right)$ are lattices in $G_{S^{\prime}} \times \prod_{w \in S-S^{\prime}} H_{w}$, the containment $\boldsymbol{G}\left(\mathcal{O}_{S^{\prime}}\right) \leq \Gamma_{S^{\prime}}$ is of finite index. Therefore, the Hausdorff distance between

$$
\Gamma_{S^{\prime}}\left(D_{\infty} \times \prod_{w \in S^{\prime}-V_{K}^{\infty}} D_{w} \times \prod_{w \in S-S^{\prime}}\left\{x_{w}\right\}\right)
$$

and

$$
\Omega_{S^{\prime}} \times \prod_{w \in S-S^{\prime}}\left\{x_{w}\right\}=\boldsymbol{G}\left(\mathcal{O}_{S^{\prime}}\right)\left(D_{\infty} \times \prod_{w \in S^{\prime}-V_{K}^{\infty}} D_{w} \times \prod_{w \in S-S^{\prime}}\left\{x_{w}\right\}\right)
$$

is finite. Combined with (1) and (2) above, the lemma follows.

The more general form of Lemma 2.2.1 that we will use in our proof of Theorem 1.4.1 is the following lemma. We will use the notation of $x_{S-S^{\prime}}$ for the point $\left(x_{w}\right)_{w \in S-S^{\prime}} \in X_{S-S^{\prime}}$.

Lemma 2.2.2. Suppose that $V_{K}^{\infty} \subseteq S^{\prime} \subseteq S$. If $y \in X_{S-S^{\prime}}$ and $y \in \boldsymbol{G}\left(\mathcal{O}_{S}\right) x_{S-S^{\prime}}$, then the Hausdorff distance between

$$
\Omega_{S} \cap\left(X_{S^{\prime}} \times\{y\}\right)
$$

and

$$
\Omega_{S^{\prime}} \times\{y\}
$$

is finite. 
Remark. Our assumption in Lemma 2.2.2 that $y \in \boldsymbol{G}\left(\mathcal{O}_{S}\right) x_{S-S^{\prime}}$ is not a serious restriction over the assumption that $y \in X_{S-S^{\prime}}$. Indeed, $\boldsymbol{G}\left(\mathcal{O}_{S}\right)$ is dense in $G_{S-S^{\prime}}$, so the orbit $\boldsymbol{G}\left(\mathcal{O}_{S}\right) x_{S-S^{\prime}}$ is a finite Hausdorff distance from the space $X_{S-S^{\prime}}$.

Proof. Let $g \in \boldsymbol{G}\left(\mathcal{O}_{S}\right)$ be such that $y=g x_{S-S^{\prime}}$. Then

$$
\begin{aligned}
\left\{h \in \boldsymbol{G}\left(\mathcal{O}_{S}\right) \mid h x_{S-S^{\prime}}=y\right\} & =g\left\{h \in \boldsymbol{G}\left(\mathcal{O}_{S}\right) \mid h x_{S-S^{\prime}}=x_{S-S^{\prime}}\right\} \\
& =g\left(\boldsymbol{G}\left(\mathcal{O}_{S}\right) \cap\left(G_{S^{\prime}} \times \prod_{w \in S-S^{\prime}} H_{w}\right)\right) \\
& =g \Gamma_{S^{\prime}},
\end{aligned}
$$

where $H_{w}$ and $\Gamma_{S^{\prime}}$ are as in the proof of the previous lemma.

Now by our choice of the points $x_{w} \in X_{w}$ for $w \in S-V_{K}^{\infty}$ at the beginning of this section, we have

$$
\begin{aligned}
\Omega_{S} \cap\left(X_{S^{\prime}} \times\{y\}\right) & =\boldsymbol{G}\left(\mathcal{O}_{S}\right)\left(D_{\infty} \times \prod_{w \in S-V_{K}^{\infty}} D_{w}\right) \cap\left(X_{S^{\prime}} \times\{y\}\right) \\
& =g \Gamma_{S^{\prime}}\left(D_{\infty} \times \prod_{w \in S^{\prime}-V_{K}^{\infty}} D_{w}\right) \times\{y\} .
\end{aligned}
$$

Notice that the final space from the above chain of equalities is a finite Hausdorff distance from

$$
g \boldsymbol{G}\left(\mathcal{O}_{S^{\prime}}\right)\left(D_{\infty} \times \prod_{w \in S^{\prime}-V_{K}^{\infty}} D_{w}\right) \times\{y\}
$$

since $\Gamma_{S^{\prime}}$ is commensurable with $\boldsymbol{G}\left(\mathcal{O}_{S^{\prime}}\right)$.

Because $g$ commensurates $\boldsymbol{G}\left(\mathcal{O}_{S^{\prime}}\right)$, the above space is also a finite Hausdorff distance from $\Omega_{S^{\prime}} \times\{y\}$.

2.3. Extending quasi-isometries of $\boldsymbol{\Omega}_{\boldsymbol{S}}$ to $\boldsymbol{X}_{\boldsymbol{S}}$. Applying Lemma 2.1.1, we can regard our quasi-isometry $\phi: \boldsymbol{G}\left(\mathcal{O}_{S}\right) \rightarrow \boldsymbol{G}\left(\mathcal{O}_{S}\right)$ as a quasi-isometry of $\Omega_{S}$. Our goal is to show that $\phi$ is equivalent to an element of $\operatorname{Comm}_{\mathrm{Aut}\left(G_{S}\right)}\left(\boldsymbol{G}\left(\mathcal{O}_{S}\right)\right)$, and we begin by extending $\phi$ to a quasi-isometry of $X_{S}$.

Lemma 2.3.1. There is a permutation of $S$, which we name $\tau$, and there are quasiisometries

$$
\phi_{v}: X_{v} \rightarrow X_{\tau(v)}
$$

such that the restriction of the quasi-isometry

$$
\phi_{S}=\prod_{v \in S} \phi_{v}: X_{S} \rightarrow X_{S}
$$

to $\Omega_{S}$ is equivalent to $\phi$. If $X_{v}$ is a higher rank space for any $v \in S$, then $\phi_{v}$ may be taken to be an isometry. 
Proof. By Proposition 6.9 of [Wo1], the quasi-isometry $\phi: \Omega_{S} \rightarrow \Omega_{S}$ extends to a quasi-isometry of $X$. That is there is some quasi-isometry $\bar{\phi}: X \rightarrow X$ such that $\left.\phi \sim \bar{\phi}\right|_{\Omega_{S}}$ where $\left.\bar{\phi}\right|_{\Omega_{S}}$ is the restriction of $\bar{\phi}$ to $\Omega_{S}$.

The map $\bar{\phi}$ preserves factors in the boundary of $X$ and an argument of Eskin's Proposition 10.1 of [Es] - can be directly applied to show that $\bar{\phi}$ is equivalent to a product of quasi-isometries of the factors of $X$, up to permutation of factors.

Note that the statement of Proposition 10.1 from [Es] claims that $X_{v}$ and $X_{\tau(v)}$ are isometric for $v \in V_{K}^{\infty}$. This is because quasi-isometric symmetric spaces are isometric up to scale.

2.4. Example of proof to come. Before continuing with the general proof, we shall pause for a moment to demonstrate the utility of Lemmas 2.2.2 and 2.3.1 by proving the following special case of Theorem 1.4.1.

Theorem 2.4.1. If $m \in \mathbb{N}$ and $m \neq 1$, then

$$
Q \mathcal{I}\left(\mathrm{PGL}_{2}(\mathbb{Z}[1 / m])\right) \cong \mathrm{PGL}_{2}(\mathbb{Q}) .
$$

Proof. Let $K=\mathbb{Q}, \boldsymbol{G}=\mathrm{PGL}_{2}$, and let $S=\left\{v_{\infty}\right\} \cup\left\{v_{p}\right\}_{p \mid m}$, where $v_{\infty}$ is the Archimedean valuation and $v_{p}$ is the $p$-adic valuation. Therefore, $\boldsymbol{G}\left(\mathcal{O}_{S}\right)=$ $\mathrm{PGL}_{2}(\mathbb{Z}[1 / m])$, the space $X_{v_{\infty}}$ is the hyperbolic plane, and $X_{v_{p}}$ is a $(p+1)$-valent regular tree.

If $\phi: \mathrm{PGL}_{2}(\mathbb{Z}[1 / m]) \rightarrow \mathrm{PGL}_{2}(\mathbb{Z}[1 / m])$ is a quasi-isometry, then by Lemma 2.3.1 we can replace $\phi$ by a quasi-isometry $\phi_{S}$ which is the product of quasiisometries

$$
\phi_{\infty}: X_{v_{\infty}} \rightarrow X_{v_{\infty}} \quad \text { and } \quad \phi_{p}: X_{v_{p}} \rightarrow X_{v_{\tau(p)}}
$$

for some permutation $\tau$ of the primes dividing $m$.

If $m$ is prime, then this theorem reduces to Taback's theorem [Ta]. Now suppose that $\ell$ is a prime dividing $m$. We let $S^{\prime}=\left\{v_{\infty}, v_{\ell}\right\}$ and $S^{\prime \prime}=\left\{v_{\infty}, v_{\tau(\ell)}\right\}$.

It follows from the density of $\operatorname{PGL}_{2}(\mathbb{Z}[1 / m])$ in $\prod_{p \mid m ; p \neq \tau(\ell)} \operatorname{PGL}_{2}\left(\mathbb{Q}_{p}\right)$ that any point in $X_{S-S^{\prime \prime}}$ is a uniformly bounded distance from the orbit $\mathrm{PGL}_{2}(\mathbb{Z}[1 / m]) x_{S-S^{\prime \prime}}$. Therefore we may assume that there is some $y \in \mathrm{PGL}_{2}(\mathbb{Z}[1 / m]) x_{S}-S^{\prime \prime}$ such that $\phi_{S}\left(X_{S^{\prime}} \times\left\{x_{S-S^{\prime}}\right\}\right) \subseteq X_{S^{\prime \prime}} \times\{y\}$.

Since $\phi\left(\Omega_{S}\right) \subseteq \Omega_{S}$, we may assume that $\phi_{S}\left(\Omega_{S} \cap\left(X_{S^{\prime}} \times\left\{x_{S-S^{\prime}}\right\}\right)\right) \subseteq \Omega_{S} \cap$ $\left(X_{S^{\prime \prime}} \times\{y\}\right)$. It follows from Lemma 2.2.2 that $\phi_{S}$ restricts to a quasi-isometry between $\Omega_{S^{\prime}} \times\left\{x_{S-S^{\prime}}\right\}$ and $\Omega_{S^{\prime \prime}} \times\{y\}$.

Note that by the product structure of $\phi_{S}$, we can assume that $\phi_{\infty} \times \phi_{\ell}$ restricts to a quasi-isometry between $\Omega_{S^{\prime}}$ and $\Omega_{S^{\prime \prime}}$ - or by Lemma 2.1.1- a quasi-isometry between $\mathrm{PGL}_{2}(\mathbb{Z}[1 / \ell])$ and $\mathrm{PGL}_{2}(\mathbb{Z}[1 / \tau(\ell)])$. Taback showed that for any such quasi-isometry we must have $\ell=\tau(\ell)$ and that $\phi_{\infty} \times \phi_{\ell}$ is equivalent to a commensurator $g_{\infty} \times g_{\ell} \in \mathrm{PGL}_{2}(\mathbb{R}) \times \mathrm{PGL}_{2}\left(\mathbb{Q}_{\ell}\right)$ where $g_{\infty}$ and $g_{\ell}$ must necessarily be included in, and represent the same element of, $\mathrm{PGL}_{2}(\mathbb{Q})[\mathrm{Ta}]$. 
As the above paragraph is independent of the prime $\ell \mid m$, the element $g_{\infty} \in$ $\mathrm{PGL}_{2}(\mathbb{Q})$ determines $\phi_{S}$ and thus $\phi$.

Having concluded our proof of the above special case, we return to the general proof. Our goal is to show that the quasi-isometry $\phi_{S}$ is equivalent to an element of $\operatorname{Comm}_{\mathrm{Aut}\left(G_{S}\right)}\left(\boldsymbol{G}\left(\mathcal{O}_{S}\right)\right)$. At this point, the proof breaks into two cases.

2.5. Case 1: $G_{V_{K}^{\infty}}$ is not locally isomorphic to $\operatorname{PGL}_{2}(\mathbb{R})$. We observe that $\operatorname{Comm}_{\text {Aut }\left(G_{S}\right)}\left(\boldsymbol{G}\left(\mathcal{O}_{S}\right)\right)$ acts by isometries on $X_{S}$. So a good first step toward our goal is to show that $\phi_{S}$ is equivalent to an isometry. First, we will show that $\phi_{V_{K}^{\infty}}$ is equivalent to an isometry.

Lemma 2.5.1. The quasi-isometry $\phi_{V_{K}^{\infty}}: X_{V_{K}^{\infty}} \rightarrow X_{V_{K}^{\infty}}$ is equivalent to an isometry of the symmetric space $X_{V_{K}^{\infty}}$. Indeed, it is equivalent to an element of $\operatorname{Comm}_{\mathrm{Aut}\left(G_{S}\right)}(\boldsymbol{G}(\mathcal{O}))$.

Proof. Notice that $\phi_{V_{K}^{\infty}}$ is simply the restriction of $\phi_{S}$ to $X_{V_{K}^{\infty}} \times\left\{x_{S-V_{K}^{\infty}}\right\}$.

Since $\boldsymbol{G}\left(\mathcal{O}_{S}\right)$ is dense in $G_{S-V_{K}^{\infty}}$, the Hausdorff distance between $\boldsymbol{G}\left(\mathcal{O}_{S}\right) x_{S-V_{K}^{\infty}}$ and $X_{S-V_{K}^{\infty}}$ is finite. Thus, by replacing $\phi_{S}$ with an equivalent quasi-isometry, we may assume that $X_{V_{K}^{\infty}} \times\left\{x_{S-V_{K}^{\infty}}\right\}$ is mapped by $\phi_{S}$ into a space $X_{V_{K}^{\infty}} \times\{y\}$ for some $y \in X_{S-V_{K}^{\infty}}$ with $y \in \boldsymbol{G}\left(\mathcal{O}_{S}\right) x_{S-V_{K}^{\infty}}$.

Since the Hausdorff distance between $\phi_{S}\left(\Omega_{S}\right)$ and $\Omega_{S}$ is finite, we have by Lemmas 2.2.2 and 2.1.1 that $\phi_{V_{K}^{\infty}}$ induces a quasi-isometry of $\boldsymbol{G}(\mathcal{O})$. The lemma follows from the existing quasi-isometric classification of arithmetic lattices using our assumption in this Case that $G_{V_{K}^{\infty}}$ is not locally isomorphic to $\mathrm{PGL}_{2}(\mathbb{R})$; see $[\mathrm{Fa}]$.

At this point, it is not difficult to see that Theorem 1.4.1 holds in the case when every non-Archimedean factor of $G_{S}$ is higher rank:

Lemma 2.5.2. If $\operatorname{rank}_{K_{v}}(\boldsymbol{G}) \geq 2$ for all $v \in S-V_{K}^{\infty}$ then $\phi_{S}$ is equivalent to an element of $\operatorname{Comm}_{\mathrm{Aut}\left(G_{S}\right)}\left(\boldsymbol{G}\left(\mathcal{O}_{S}\right)\right)$.

Proof. By Lemma 2.3.1, $\phi_{v}$ is equivalent to an isometry for all $v \in S-V_{K}^{\infty}$. Combined with Lemma 2.5.1, we know that $\phi_{S}$ is equivalent to an isometry.

That $\phi_{S}$ is equivalent to an element of $\operatorname{Comm}_{\text {Aut }\left(G_{S}\right)}\left(\boldsymbol{G}\left(\mathcal{O}_{S}\right)\right)$ follows from Proposition 7.2 of [Wo1]. Indeed, any isometry of $X_{S}$ that preserves $\Omega_{S}$ up to finite Hausdorff distance corresponds in a natural way to an automorphism of $G_{S}$ that preserves $\boldsymbol{G}\left(\mathcal{O}_{S}\right)$ up to finite Hausdorff distance, and any such automorphism of $G_{S}$ is shown in Proposition 7.2 of [Wo1] to be a commensurator.

For the remainder of Case 1, we are left to assume that there is at least one $w \in S-V_{K}^{\infty}$ such that $\operatorname{rank}_{K_{w}}(\boldsymbol{G})=1$. 
Before beginning the proof of the next and final lemma for Case 1, it will be best to recall some standard facts about boundaries.

Tree boundaries. If $w$ is a non-Archimedean valuation of $K$, and $\operatorname{rank}_{K_{w}}(\boldsymbol{G})=1$, then $X_{w}$ is a tree.

For any minimal $K_{w}$-parabolic subgroup of $\boldsymbol{G}$, say $\boldsymbol{M}$, we let $\varepsilon_{\boldsymbol{M}}$ be the end of $X_{w}$ such that $\boldsymbol{M}\left(K_{w}\right) \varepsilon_{\boldsymbol{M}}=\varepsilon_{\boldsymbol{M}}$. Notice that the space of all ends of the form $\varepsilon_{\boldsymbol{P}}$ where $\boldsymbol{P}$ is a minimal $K$-parabolic subgroup of $\boldsymbol{G}$ forms a dense subset of the space of ends of $X_{w}$.

Tits boundaries. For any minimal $K$-parabolic subgroup of $\boldsymbol{G}$, say $\boldsymbol{P}$, we let $\delta_{\boldsymbol{P}}$ be the simplex in the Tits boundary of $X_{V_{K}^{\infty}}$ corresponding to the group $\prod_{v \in V_{K}^{\infty}} \boldsymbol{P}\left(K_{v}\right)$.

If $\delta$ is a simplex in the Tits boundary of $X_{V_{K}^{\infty}}$, and $\varepsilon$ is an end of the tree $X_{w}$, then we denote the join of $\delta$ and $\varepsilon$ by $\delta * \varepsilon$. It is a simplex in the Tits boundary of $X_{T}$ where $T=V_{K}^{\infty} \cup\{w\}$.

Lemma 2.5.3. Let $w \in S-V_{K}^{\infty}$ be such that $\operatorname{rank}_{K_{w}}(\boldsymbol{G})=1$. Then $\phi_{w}: X_{w} \rightarrow$ $X_{\tau(w)}$ is equivalent to an isometry that is induced by an isomorphism of topological groups $\boldsymbol{G}\left(K_{w}\right) \rightarrow \boldsymbol{G}\left(K_{\tau(w)}\right)$.

Proof. Below we will denote the set of valuations $V_{K}^{\infty} \cup\{\tau(w)\}$ by $T^{\tau}$.

We begin by choosing a minimal $K$-parabolic subgroup of $\boldsymbol{G}$, say $\boldsymbol{P}$, and a geodesic ray $\rho: \mathbb{R}_{\geq 0} \rightarrow X_{T}$ that limits to the interior of the simplex $\delta_{\boldsymbol{P}} * \varepsilon_{\boldsymbol{P}}$.

By Lemma 2.5.1, the image of $\phi_{T} \circ \rho$ under the projection $X_{T} \rightarrow X_{V_{K}^{\infty}}$ limits to a point in the interior of $\delta_{\boldsymbol{Q}}$ for some minimal $K$-parabolic subgroup $\boldsymbol{Q}$. Similarly, $\phi_{w}$ is a quasi-isometry of a tree, so it maps each geodesic ray into a bounded neighborhood of a geodesic ray that is unique up to finite Hausdorff distance. Thus, the image of $\phi_{T} \circ \rho$ under the projection $X_{T} \rightarrow X_{\tau(w)}$ limits to $\varepsilon_{\mathbf{Q}^{\prime}}$ for some minimal $K_{\tau(w)^{-}}$ parabolic subgroup $\mathbf{Q}^{\prime}$. Together, these results imply that $\phi_{T} \circ \rho$ is a finite Hausdorff distance from a geodesic ray that limits to a point in the interior of $\delta_{\boldsymbol{Q}} * \varepsilon_{\mathbf{Q}^{\prime}}$.

By Lemma 3.4.1, there is a subspace $\mathscr{H}_{\boldsymbol{P}}$ of $X_{T}$ corresponding to $\boldsymbol{P}$ (called a "T-horoball") such that

$$
t \mapsto d\left(\rho(t), X_{T}-\mathscr{H}_{\boldsymbol{P}}\right)
$$

is an unbounded function. Thus,

$$
t \mapsto d\left(\phi_{T} \circ \rho(t), X_{T \tau}-\phi_{T}\left(\mathscr{H}_{\boldsymbol{P}}\right)\right)
$$

is also unbounded.

Using Lemma 3.2.1 and the fact that $\phi_{T}\left(\Omega_{T}\right)$ is a finite Hausdorff distance from $\Omega_{T^{\tau}}$, we may replace $\phi_{T}$ with an equivalent quasi-isometry to deduce that $\phi_{T}\left(\mathcal{H}_{\boldsymbol{P}}\right)$ is contained in the union of all $T^{\tau}$-horoballs in $X_{T^{\tau}}$.

It will be clear from the definition given in Section 3 that each $T$-horoball is connected. In addition, Lemma 3.2.2 states that the collection of $T^{\tau}$-horoballs is 
pairwise disjoint, so it follows that $\phi_{T}\left(\mathscr{H}_{\boldsymbol{P}}\right)$ is a finite distance from a single $T^{\tau}$ horoball $\mathscr{H}_{\boldsymbol{M}} \subseteq X_{T^{\tau}}$ where $\boldsymbol{M}$ is a minimal $K$-parabolic subgroup of $\boldsymbol{G}$. Therefore,

$$
t \mapsto d\left(\phi_{T} \circ \rho(t), X_{T^{\tau}}-\mathscr{H}_{\boldsymbol{M}}\right)
$$

is unbounded.

Because the above holds for all $\rho$ limiting to $\delta_{\boldsymbol{P}} * \varepsilon_{\boldsymbol{P}}$, and because $\phi_{T} \circ \rho$ limits to $\delta \boldsymbol{Q} * \varepsilon_{\mathbf{Q}^{\prime}}$, we have by Lemma 3.4.2 that $\boldsymbol{Q}=\boldsymbol{M}=\mathbf{Q}^{\prime}$. That is, $\phi_{V_{K}^{\infty}}$ completely determines the map that $\phi_{w}$ induces between the ends of the trees $X_{w}$ and $X_{\tau(w)}$ that correspond to $K$-parabolic subgroups of $\boldsymbol{G}$.

Recall that by Lemma 2.5.1, $\phi_{V_{K}^{\infty}}$ is equivalent to a commensurator of $\boldsymbol{G}(\mathcal{O}) \leq$ $G_{V_{K}^{\infty}}$. Using Lemma 7.3 of [Wo1], $\phi_{V_{K}^{\infty}}$ (regarded as an automorphism of $\left.G_{V_{K}^{\infty}}\right)$ restricts to $\boldsymbol{G}(K)$ as a composition

$$
\delta \circ \sigma^{\circ}: \boldsymbol{G}(K) \rightarrow \boldsymbol{G}(K)
$$

where $\sigma$ is an automorphism of $K$,

$$
\sigma^{\circ}: \boldsymbol{G}(K) \rightarrow{ }^{\sigma} \boldsymbol{G}(K)
$$

is the map obtained by applying $\sigma$ to the entries of elements in $\boldsymbol{G}(K)$, and

$$
\delta:{ }^{\sigma} \boldsymbol{G} \rightarrow \boldsymbol{G}
$$

is a $K$-isomorphism of $K$-groups.

Thus, if $\partial X_{w}$ and $\partial X_{\tau(w)}$ are the space of ends of the trees $X_{w}$ and $X_{\tau(w)}$ respectively, and if $\partial \phi_{w}: \partial X_{w} \rightarrow \partial X_{\tau(w)}$ is the boundary map induced by $\phi_{w}$, then we have shown that

$$
\partial \phi_{w}\left(\varepsilon_{\boldsymbol{P}}\right)=\varepsilon_{\delta}(\sigma \boldsymbol{P})
$$

for any $\boldsymbol{P} \leq \boldsymbol{G}$ that is a minimal $K$-parabolic subgroup of $\boldsymbol{G}$.

Our next goal is to show that the valuation $w \circ \sigma^{-1}$ is equivalent to $\tau(w)$. If this is the case, then $\delta \circ \sigma^{\circ}$ extends from a group automorphism of $\boldsymbol{G}(K)$ to a topological group isomorphism

$$
\alpha_{w}: \boldsymbol{G}\left(K_{w}\right) \rightarrow \boldsymbol{G}\left(K_{\tau(w)}\right) .
$$

If $\partial \alpha_{w}: \partial X_{w} \rightarrow \partial X_{\tau(w)}$ is the map induced by $\alpha_{w}$, then $\partial \alpha_{w}$ equals $\partial \phi_{w}$ on the subset of ends in $\partial X_{w}$ corresponding to $K$-parabolic subgroups of $\boldsymbol{G}$ since $\alpha_{w}$ extends $\delta \circ \sigma^{\circ}$. Therefore, $\partial \alpha_{w}=\partial \phi_{w}$ on all of $\partial X_{w}$ by the density of the " $K$-rational ends" in $\partial X_{w}$. Thus, $\alpha_{w}$ determines $\phi_{w}$ up to equivalence. This would prove our lemma.

So to finish the proof of this lemma, we will show that $w \circ \sigma^{-1}$ is equivalent to $\tau(w)$.

For any maximal $K$-split torus $\boldsymbol{S} \leq \boldsymbol{G}$, we let $\gamma_{\boldsymbol{S}}^{w} \subseteq X_{w}$ (resp. $\left.\gamma_{\boldsymbol{S}}^{\tau(w)} \subseteq X_{\tau(w)}\right)$ be the geodesic that $\boldsymbol{S}\left(K_{w}\right)$ (resp. $\left.\boldsymbol{S}\left(K_{\tau(w)}\right)\right)$ acts on by translations.

Fix $\boldsymbol{S}$ and $\boldsymbol{T}$, two maximal $K$-split tori in $\boldsymbol{G}$ such that $\gamma_{\boldsymbol{S}}^{w} \cap \gamma_{\boldsymbol{T}}^{w}$ is nonempty and bounded. We choose a point $a \in \gamma_{\boldsymbol{S}}^{w} \cap \gamma_{\boldsymbol{T}}^{w}$. 
Since $\boldsymbol{S}\left(\mathcal{O}_{T}\right)$ is dense in $\boldsymbol{S}\left(K_{w}\right)$, there exists a group element $g_{n} \in \boldsymbol{S}\left(\mathcal{O}_{T}\right)$ for each $n \in \mathbb{N}$ such that

$$
d\left(g_{n}\left(\gamma_{\boldsymbol{T}}^{w}\right), a\right)>n .
$$

Note that $g_{n}\left(\gamma_{\boldsymbol{T}}^{w}\right)=\gamma_{g_{n} \boldsymbol{T} g_{n}^{-1}}^{w}$. Thus

$$
d\left(\phi_{w}\left(\gamma_{g_{n} \boldsymbol{T} g_{n}^{-1}}^{w}\right), \phi_{w}(a)\right)
$$

is an unbounded sequence. from

As $g_{n} \boldsymbol{T} g_{n}^{-1}$ is $K$-split, $\phi_{w}\left(\gamma_{g_{n}}^{w} \boldsymbol{T} g_{n}^{-1}\right)$ is a uniformly bounded Hausdorff distance

$$
\gamma_{\delta \circ \sigma^{\circ}\left(g_{n} \boldsymbol{T} g_{n}^{-1}\right)}^{\tau(w)}=\delta \circ \sigma^{\circ}\left(g_{n}\right) \gamma_{\delta \circ \sigma^{\circ}(\boldsymbol{T})}^{\tau(w)}
$$

because a geodesic in $X_{\tau(w)}$ is determined by its two ends.

We finally have that

$$
d\left(\delta \circ \sigma^{\circ}\left(g_{n}\right) \gamma_{\delta \circ \sigma^{\circ}(\boldsymbol{T})}^{\tau(w)}, \phi_{w}(a)\right)
$$

is an unbounded sequence. It is this statement that we shall contradict by assuming that $w \circ \sigma^{-1}$ is inequivalent to $\tau(w)$.

Note that $g_{n} \in \boldsymbol{G}\left(\mathcal{O}_{v}\right)$ for all $v \in V_{K}-T$ since $g_{n} \in \boldsymbol{S}\left(\mathcal{O}_{T}\right)$. Thus, $\sigma^{0}\left(g_{n}\right) \in$ ${ }^{\sigma} \boldsymbol{G}\left(\mathcal{O}_{v \circ \sigma^{-1}}\right)$ for all $v \in V_{K}-T$. If it were the case that $w \circ \sigma^{-1}$ is inequivalent to $\tau(w)$, then it follows that $\sigma^{0}\left(g_{n}\right) \in{ }^{\sigma} \boldsymbol{G}\left(\mathcal{O}_{\tau(w)}\right)$. Hence, $\delta \circ \sigma^{0}\left(g_{n}\right)$ defines a bounded sequence in $\boldsymbol{G}\left(K_{\tau(w)}\right)$. Therefore,

$$
d\left(\delta \circ \sigma^{\circ}\left(g_{n}\right) \gamma_{\delta \circ \sigma^{\circ}(\boldsymbol{T})}^{\tau(w)}, \phi_{w}(a)\right)
$$

is a bounded sequence, our contradiction.

The proof of Theorem 1.4.1 in Case 1 is complete with the observation that applications of Lemma 2.5.3 to tree factors, allows us to apply the Proposition 7.2 of [Wo1] as we did in Lemma 2.5.2.

2.6. Case 2: $\boldsymbol{G}_{V_{K}^{\infty}}$ is locally isomorphic to $\mathbf{P G L}_{2}(\mathbb{R})$. It follows that $V_{K}^{\infty}$ contains a single valuation $v$, and that $K_{v} \cong \mathbb{R}$. Thus $K=\mathbb{Q}$, and $V_{K}^{\infty}$ is the set containing only the standard real metric on $\mathbb{Q}$.

Our assumption that $\boldsymbol{G}$ is absolutely simple implies that $G_{V_{K}^{\infty}}$ is actually isomorphic to $\mathrm{PGL}_{2}(\mathbb{R})$. Thus, $\boldsymbol{G}$ is a $\mathbb{Q}$-form of $\mathrm{PGL}_{2}$. As we are assuming that $\boldsymbol{G}$ is $\mathbb{Q}$-isotropic, it follows from the classification of $\mathbb{Q}$-forms of $\mathrm{PGL}_{2}$ that $\boldsymbol{G}$ and $\mathrm{PGL}_{2}$ are $\mathbb{Q}$-isomorphic (see e.g. p. 55 of [Ti]).

From our assumptions in the statement of Theorem 1.4.1, $S \neq V_{K}^{\infty}$. As the only valuations, up to scale, on $\mathbb{Q}$ are the real valuation and the $p$-adic valuations, $\boldsymbol{G}\left(\mathcal{O}_{S}\right)$ is commensurable with $\operatorname{PGL}_{2}(\mathbb{Z}[1 / m])$ for some $m \in \mathbb{N}$ with $m \neq 1$. Thus, Case 2 of Theorem 1.4.1 follows from Theorem 2.4.1.

Our proof of Theorem 1.4.1 is complete modulo the material from Section 3. 


\section{Horoball patterns in a product of a tree and a symmetric space}

In this section we will study the components of $X_{S}-\Omega_{S}$ when $X_{S}$ is a product of a symmetric space and a tree.

Setting notation. Suppose that $w$ is a non-Archimedean valuation on $K$ such that $\operatorname{rank}_{K_{w}}(\boldsymbol{G})=1$. Then set $T$ equal to $V_{K}^{\infty} \cup\{w\}$.

3.1. Horoballs in rank one symmetric spaces. Let $\boldsymbol{P}$ be a minimal $K$-parabolic subgroup of $\boldsymbol{G}$. As in the previous section, we let $\delta_{\boldsymbol{P}}$ be the simplex in the Tits boundary of $X_{V_{K}^{\infty}}$ corresponding to the group $\prod_{v \in V_{K}^{\infty}} \boldsymbol{P}\left(K_{v}\right)$.

Notice that $\boldsymbol{G}$ being $K$-isotropic and $\operatorname{rank}_{K_{w}}(\boldsymbol{G})=1$ together imply that $\operatorname{rank}_{K}(\boldsymbol{G})=1$. Borel proved that the latter equality implies that $X_{V_{K}^{\infty}}-\Omega_{V_{K}^{\infty}}$ can be taken to be a disjoint collection of horoballs ([Bo], 17.10).

To any horoball of $X_{V_{K}^{\infty}}-\Omega_{V_{K}^{\infty}}$, say $H$, there corresponds a unique $\delta_{\boldsymbol{P}}$ as above such that any geodesic ray $\rho: \mathbb{R}_{\geq 0} \rightarrow X_{V_{K}^{\infty}}$ that limits to $\delta_{\boldsymbol{P}}$ defines an unbounded function

$$
t \mapsto d\left(\rho(t), X_{V_{K}^{\infty}}-H\right) .
$$

3.2. $\boldsymbol{T}$-horoballs in $\boldsymbol{X}_{\boldsymbol{T}}$. Let $y \in X_{w}$ and suppose $y \in \boldsymbol{G}\left(\mathcal{O}_{T}\right) x_{w}$. Recall that by Lemma 2.2.2 the space $\Omega_{T} \cap\left(X_{V_{K}^{\infty}} \times\{y\}\right)$ is a finite Hausdorff distance from $\Omega_{V_{K}^{\infty}} \times\{y\}$.

For any minimal $K$-parabolic subgroup of $\boldsymbol{G}$, say $\boldsymbol{P}$, we let $\mathscr{H}_{\boldsymbol{P}, \infty}^{y} \subseteq X_{V_{K}^{\infty}} \times\{y\}$ be the horoball of $\Omega_{T} \cap\left(X_{V_{K}^{\infty}} \times\{y\}\right)$ that corresponds to $\delta_{\boldsymbol{P}}$.

For arbitrary $x \in X_{w}$, we define

$$
\mathscr{H}_{\boldsymbol{P}, \infty}^{x}=\mathscr{H}_{\boldsymbol{P}, \infty}^{y}
$$

where $y \in \boldsymbol{G}\left(\mathcal{O}_{T}\right) x_{w}$ minimizes the distance between $x$ and $\boldsymbol{G}\left(\mathcal{O}_{T}\right) x_{w}$.

We let

$$
\mathscr{H}_{\boldsymbol{P}}=\bigcup_{x \in X_{w}}\left(\mathscr{H}_{\boldsymbol{P}, \infty}^{x} \times\{x\}\right) .
$$

Each of the spaces $\mathscr{H}_{\boldsymbol{P}}$ is called a $T$-horoball.

Let $\mathcal{P}$ be the set of all minimal $K$-parabolic subgroups of $\boldsymbol{G}$. The following lemma follows directly from our definitions. It will be used in the proof of Lemma 2.5.3.

Lemma 3.2.1. The Hausdorff distance between $X_{T}-\Omega_{T}$ and $\bigcup_{\boldsymbol{P} \in \mathcal{P}} \mathcal{H}_{\boldsymbol{P}}$ is finite.

We record another observation to be used in the proof of Lemma 2.5.3.

Lemma 3.2.2. If $\boldsymbol{P} \neq \boldsymbol{Q}$ are minimal $K$-parabolic subgroups of $\boldsymbol{G}$, then $\mathscr{H}_{\boldsymbol{P}} \cap \mathscr{H}_{\boldsymbol{Q}}=\emptyset$. 
Proof. The horoballs comprising $\left(X_{V_{K}^{\infty}}-\Omega_{V_{K}^{\infty}}\right) \times\left\{x_{w}\right\}$ are pairwise disjoint, and are a finite Hausdorff distance from the horoballs of $\Omega_{T} \cap\left(X_{V_{K}^{\infty}} \times\left\{x_{w}\right\}\right)$ by Lemma 2.2.1. Hence, if $y=g x_{w}$ for some $g \in \boldsymbol{G}\left(\mathcal{O}_{T}\right)$, then the horoballs determined by

$$
\Omega_{T} \cap\left(X_{V_{K}^{\infty}} \times\{y\}\right)=g\left[\Omega_{T} \cap\left(X_{V_{K}^{\infty}} \times\left\{x_{w}\right\}\right)\right]
$$

are disjoint.

3.3. Deformations of horoballs over geodesics in $\boldsymbol{X}_{\boldsymbol{w}}$. We let $\pi: X_{T} \rightarrow X_{V_{K}^{\infty}}$ be the projection map. Note that if $x \in X_{w}$ and $\boldsymbol{P}$ is a minimal $K$-parabolic subgroup of $\boldsymbol{G}$, then $\pi\left(\mathcal{H}_{\boldsymbol{P}, \infty}^{x}\right)$ is a horoball in $X_{V_{K}^{\infty}}$ that is based at $\delta_{\boldsymbol{P}}$.

Recall that for any minimal $K_{w}$-parabolic subgroup of $\boldsymbol{G}$, say $\boldsymbol{Q}$, we denote the point in the boundary of the tree $X_{w}$ that corresponds to $\boldsymbol{Q}\left(K_{w}\right)$ by $\varepsilon \boldsymbol{Q}$.

Lemma 3.3.1. Suppose that $\boldsymbol{P}$ is a minimal $K$-parabolic subgroup of $\boldsymbol{G}$ and that $\boldsymbol{Q}$ is a minimal $K_{w}$-parabolic subgroup of $\boldsymbol{G}$. If $\gamma: \mathbb{R} \rightarrow X_{w}$ is a geodesic with $\gamma(\infty)=\varepsilon_{\boldsymbol{P}}$ and $\gamma(-\infty)=\varepsilon_{\mathbf{Q}}$ then

(i) $s \leq t$ implies $\pi\left(\mathscr{H}_{\boldsymbol{P}, \infty}^{\gamma(s)}\right) \subseteq \pi\left(\mathscr{H}_{\boldsymbol{P}, \infty}^{\gamma(t)}\right)$,

(ii) $\bigcup_{t \in \mathbb{R}} \pi\left(\mathscr{H}_{\boldsymbol{P}, \infty}^{\gamma(t)}\right)=X_{V_{K}^{\infty}}$,

(iii) $\bigcap_{t \in \mathbb{R}}\left(\mathscr{H}_{\boldsymbol{P}, \infty}^{\gamma(t)}\right)=\emptyset$,

(iv) there exists constants $L_{\boldsymbol{P}}, C>0$ such that if

$$
h(s, t)=d\left(\pi\left(\mathscr{H}_{\boldsymbol{P}, \infty}^{\gamma(s)}\right), \pi\left(\mathcal{H}_{\boldsymbol{P}, \infty}^{\gamma(t)}\right)\right)
$$

then

$$
\left|h(s, t)-L_{\boldsymbol{P}}\right| s-t|| \leq C .
$$

Proof. As the ends of $X_{w}$ corresponding to $K$-parabolic subgroups are a dense subset of the full space of ends, it suffices to prove this lemma when $Q$ is defined over $K$. In this case, the image of $\gamma$ corresponds to a $K$-split torus $\boldsymbol{S} \leq \boldsymbol{G}$ that is contained in $\boldsymbol{P}$.

Let $\alpha$ be a root of $\boldsymbol{G}$ with respect to $\boldsymbol{S}$ such that $\alpha$ is positive in $\boldsymbol{P}$. Since the diagonal embedding of $\boldsymbol{S}\left(\mathcal{O}_{T}\right)$ in the group $\prod_{v \in V_{K}^{\infty}} \boldsymbol{S}\left(K_{v}\right)$ has a dense image, there is some $b \in \boldsymbol{S}\left(\mathcal{O}_{T}\right)$ such that $|\alpha(\boldsymbol{S}(b))|_{v}<1$ for all $v \in V_{K}^{\infty}$. Thus, $\boldsymbol{S}(b) \pi\left(\mathscr{H}_{\boldsymbol{P}, \infty}^{\gamma(0)}\right)$ is a horoball in $X_{V_{K}^{\infty}}$ that strictly contains $\pi\left(\mathcal{H}_{\boldsymbol{P}, \infty}^{\gamma(0)}\right)$. Generally, we have

$$
\boldsymbol{S}(b)^{m} \pi\left(\mathcal{H}_{\boldsymbol{P}, \infty}^{\gamma(0)}\right) \subsetneq \boldsymbol{S}(b)^{n} \pi\left(\mathcal{H}_{\boldsymbol{P}, \infty}^{\gamma(0)}\right)
$$

for all $m, n \in \mathbb{Z}$ with $m<n$.

By the product formula we have $|\alpha(S(b))|_{w}>1$. Thus, there is a positive number $\lambda>0$ such that $\gamma(n \lambda)=\boldsymbol{S}(b)^{n} \gamma(0)$ for any $n \in \mathbb{Z}$. It follows for $m<n$ that

$$
\pi\left(\mathcal{H}_{\boldsymbol{P}, \infty}^{\gamma(m \lambda)}\right)=\boldsymbol{S}(b)^{m} \pi\left(\mathcal{H}_{\boldsymbol{P}, \infty}^{\gamma(0)}\right) \subsetneq \boldsymbol{S}(b)^{n} \pi\left(\mathcal{H}_{\boldsymbol{P}, \infty}^{\gamma(0)}\right)=\pi\left(\mathcal{H}_{\boldsymbol{P}, \infty}^{\gamma(n \lambda)}\right) .
$$


We let

$$
L_{\boldsymbol{P}}=\frac{1}{\lambda} d\left(\pi\left(\mathscr{H}_{\boldsymbol{P}, \infty}^{\gamma(0)}\right), \pi\left(\mathscr{H}_{\boldsymbol{P}, \infty}^{\gamma(\lambda)}\right)\right)
$$

so that

$$
h(m \lambda, n \lambda)=L_{\boldsymbol{P}} \lambda|m-n|=L_{\boldsymbol{P}}|m \lambda-n \lambda| .
$$

Then we take

$$
C^{\prime}=\max _{0 \leq s \leq t \leq \lambda}\left\{d\left(\pi\left(\mathscr{H}_{\boldsymbol{P}, \infty}^{\gamma(s)}\right), \pi\left(\mathscr{H}_{\boldsymbol{P}, \infty}^{\gamma(t)}\right)\right)\right\}
$$

and, say,

$$
C=2 C^{\prime}+L_{\boldsymbol{P}} d(\gamma(0), \gamma(\lambda)) .
$$

3.4. Basepoints in the Tits boundary for $\boldsymbol{T}$-horoballs. The Tits boundary for $X$ is the spherical join of the Tits boundary for the symmetric space $X_{V_{K}^{\infty}}$ and the Tits boundary for the tree $X_{w}$.

The purpose of the following two lemmas - and of this entire section - is to show that each $T$-horoball $\mathscr{H}_{\boldsymbol{P}}$ is geometrically associated with the join of $\delta_{\boldsymbol{P}}$ and $\varepsilon_{\boldsymbol{P}}$, denoted $\delta_{\boldsymbol{P}} * \varepsilon_{\boldsymbol{P}}$.

Lemma 3.4.1. Let $\boldsymbol{P}$ be a minimal $K$-parabolic subgroup of $\boldsymbol{G}$. Any geodesic ray $\rho: \mathbb{R}_{\geq 0} \rightarrow X_{T}$ that limits to the simplex $\delta_{\boldsymbol{P}} * \varepsilon_{\boldsymbol{P}}$ in the Tits boundary of $X_{T}$ defines an unbounded function when composed with the distance from the complement of $\mathscr{H}_{\boldsymbol{P}}$ in $X_{T}$ :

$$
t \mapsto d\left(\rho(t), X_{T}-\mathscr{H}_{\boldsymbol{P}}\right)
$$

Proof. Any such geodesic ray $\rho$ is a product of a geodesic ray $b: \mathbb{R}_{\geq 0} \rightarrow X_{V_{K}^{\infty}}$ that limits to $\delta_{\boldsymbol{P}}$ and a geodesic ray $c: \mathbb{R}_{\geq 0} \rightarrow X_{w}$ that limits to $\varepsilon_{\boldsymbol{P}}$.

Let $Y=\pi\left(\mathscr{H}_{\boldsymbol{P}, \infty}^{c(0)}\right) \times c\left(\mathbb{R}_{\geq 0}\right)$. Since

$$
t \mapsto d\left(b(t), X_{V_{K}^{\infty}}-\pi\left(\mathscr{H}_{\boldsymbol{P}, \infty}^{c(0)}\right)\right)
$$

is unbounded, it follows that

$$
t \mapsto d\left(\rho(t), X_{T}-Y\right)
$$

is unbounded. The lemma follows from Lemma 3.3.1 (i), which guarantees that $Y \subseteq \mathscr{H}_{\boldsymbol{P}}$

Lemma 3.4.2. Suppose that $\boldsymbol{Q}$ and $\boldsymbol{M}$ are minimal $K$-parabolic subgroups of $\boldsymbol{G}$, and that $\mathbf{Q}^{\prime}$ is a minimal $K_{w}$-parabolic subgroup of $\boldsymbol{G}$ with $\boldsymbol{M} \neq \boldsymbol{Q}$ or $\boldsymbol{M} \neq \mathbf{Q}^{\prime}$. Then there is a geodesic ray $\rho: \mathbb{R}_{\geq 0} \rightarrow X$ with $\rho(\infty) \in \delta_{\boldsymbol{Q}} * \varepsilon_{\mathbf{Q}^{\prime}}$ such that the function

$$
t \mapsto d\left(\rho(t), X_{T}-\mathscr{H}_{\boldsymbol{M}}\right)
$$

is bounded. 
Proof. Choose a geodesic ray $b: \mathbb{R}_{\geq 0} \rightarrow X_{V_{K}^{\infty}}$ that limits to $\delta_{\boldsymbol{Q}}$ and a geodesic ray $c: \mathbb{R}_{\geq 0} \rightarrow X_{w}$ that limits to $\varepsilon \boldsymbol{Q}^{\prime}$. Let $r$ be the ratio of the speed of $b$ to the speed of $c$.

If $\boldsymbol{M} \neq \mathbf{Q}^{\prime}$, then after ignoring at most a bounded interval of $c$, we can extend $c$ to a bi-infinite geodesic with $c(-\infty)=\varepsilon_{\boldsymbol{M}}$. With $L_{\boldsymbol{M}}$ as in Lemma 3.3.1, $\rho(t)=$ $\left(b\left(L_{\boldsymbol{M}} t\right), c(r t)\right)$ defines a geodesic ray satisfying the lemma.

In the remaining case, $\boldsymbol{M} \neq \boldsymbol{Q}$ and $\boldsymbol{M}=\mathbf{Q}^{\prime}$.

The distance from $b(t)$ to $\pi\left(\mathscr{H}_{\boldsymbol{M}, \infty}^{b(0)}\right)$ is a convex function in $t$. Since $\boldsymbol{M} \neq \boldsymbol{Q}$, this function has a positive derivative, $u>0$, for some large value of $t$. Then $\rho(t)=\left(b\left(L_{\boldsymbol{M}} t\right), c(u r t)\right)$ defines a geodesic ray satisfying the lemma.

\section{References}

[Bo] A. Borel, Introduction aux groupes arithmétiques. Hermann, Paris 1969. Zbl 0186.33202 MR 0244260

[Es] A. Eskin, Quasi-isometric rigidity of nonuniform lattices in higher rank symmetric spaces. J. Amer. Math. Soc. 11 (1998), 321-361. Zbl 0885.22017 MR 1475886

[Fa] B. Farb, The quasi-isometry classification of lattices in semisimple Lie groups. Math. Res. Lett. 4 (1997), 705-717. Zbl 0889.22010 MR 1484701

[Fa-Sch] B. Farb and R. Schwartz, The large-scale geometry of Hilbert modular groups. $J$. Differential Geom. 44 (1996), 435-478. Zbl 0871.11035 MR 1431001

[K-L] B. Kleiner and B. Leeb, Rigidity of quasi-isometries for symmetric spaces and Euclidean buildings. Inst. Hautes Études Sci. Publ. Math. 86 (1997), 115-197. Zbl 0910.53035 MR 1608566

[Sch1] R. E. Schwartz, The quasi-isometry classification of rank one lattices. Inst. Hautes Études Sci. Publ. Math. 82 (1995), 133-168. Zbl 852.22010 MR 1383215

[Sch2] R. E. Schwartz, Quasi-isometric rigidity and Diophantine approximation. Acta Math. 177 (1996), 75-112. Zbl 1029.11038 MR 1417087

[Ta] J. Taback, Quasi-isometric rigidity for $\operatorname{PSL}_{2}(\mathbb{Z}[1 / p])$. Duke Math. J. 101 (2000), 335-357. Zbl 0954.22006 MR 1738174

[Ta-Wh] J. Taback and K. Whyte, The large-scale geometry of some metabelian groups. Michigan Math. J. 52 (2004), 205-218. Zbl 1072.20049 MR 2043406

[Ti] J. Tits, Classification of algebraic semisimple groups. In Algebraic groups and discontinuous subgroups, Proc. Symp. Pure Math. 9, Amer. Math. Soc., Providence, RI, 1966, 33-62. Zbl 0238.20052 MR 0224710

[Wo1] K. Wortman, Quasi-isometric rigidity of higher rank $S$-arithmetic lattices. Geom. Topol. 11 (2007), 995-1048. Zbl 1171.22008 MR 2326941

[Wo2] K. Wortman, Quasi-isometries of irreducible positive-rank arithmetic groups over function fields. Appendix to: M. Einsiedler and A. Mohammadi, A joining classification and a special case of Raghunathan's conjecture in positive characteristic. J. Analyse Math., to appear; arXiv:1010.5490 
Received March 13, 2008; revised December 28, 2009

K. Wortman, Department of Mathematics, University of Utah, 155 South 1400 East, Room 233, Salt Lake City, UT 84112-0090, U.S.A.

E-mail:wortman@math.utah.edu 